\title{
COMPACT ACTIONS ON C*-ALGEBRAS
}

\author{
by CHARLES A. AKEMANN† and STEVE WRIGHT
}

(Received 23 April, 1979)

1. Introduction. In Section 33 of [2], Bonsall and Duncan define an element $t$ of a Banach algebra $\mathscr{A}$ to act compactly on $\mathscr{A}$ if the map $a \rightarrow$ tat is a compact operator on $\mathscr{A}$. In this paper, the arguments and technique of [1] are used to study this question for $\mathrm{C}^{*}$-algebras (see also [10]). We determine the elements $b$ of a $C^{*}$-algebra for which the maps $a \rightarrow b a, a \rightarrow a b, a \rightarrow a b+b a, a \rightarrow b a b$ are compact (respectively weakly compact), determine the $C^{*}$-algebras which are compact in the sense of Definition 9, of [2, p. 177] and give a characterization of the ${ }^{*}$-automorphisms of $\mathscr{A}$ which are weakly compact perturbations of the identity.

We introduce the notation which will be used in the sequel. If $\boldsymbol{H}$ is a Hilbert space, $\boldsymbol{B}(\boldsymbol{H})$ and $K(H)$ denote respectively the $\mathrm{W}^{*}$-algebra of all bounded operators on $H$ and the $C^{*}$-algebra of all compact operators on $H$. A $C^{*}$-algebra $\mathscr{A}$ is said to act atomically on a Hilbert space $H$ if there exists an orthogonal family $\left\{P_{\alpha}\right\}$ of projections in $B(H)$, each commuting with $\mathscr{A}$, such that $\bigoplus_{\alpha} P_{\alpha}$ is the identity operator on $H, \mathscr{A} P_{\alpha}$, acts irreducibly on $P_{\alpha}(H)$, and $A P_{\alpha}$ is not unitarily equivalent to $A P_{\beta}$ for $\alpha \neq \beta$.

If $\left\{\mathscr{A}_{\lambda}: \lambda \in \Lambda\right\}$ is a family of $C^{*}$-algebras, the $C^{*}$-direct sum $\bigoplus_{\lambda} \mathscr{A}_{\lambda}$ of the $\mathscr{A}_{\lambda}$ 's is the $C^{*}$-algebra of all functions $f(\lambda) \in \mathscr{A}_{\lambda}, \lambda \in \Lambda$, with

$$
\|f\|=\sup \{\|f(\lambda)\|: \lambda \in \Lambda\}<\infty,
$$

equipped with pointwise operations. The restricted $C^{*}$-direct sum $\hat{\oplus}_{\lambda} \mathscr{A}_{\lambda}$ is the $C^{*}$ subalgebra of $\bigoplus_{\lambda} \mathscr{A}_{\lambda}$ consisting of all functions $f$ with $\{\lambda:\|f(\lambda)\| \geqq \varepsilon\}$ finite for all $\varepsilon>0$.

A projection $p$ of a $C^{*}$-algebra $\mathscr{A}$ is said to be finite-dimensional if $p \mathscr{A} p$ is finite-dimensional. A $C^{*}$-algebra is said to be of elementary type if it is isomorphic to $K(H)$ for some Hilbert space $H$.

By an ideal of a $\mathrm{C}^{*}$-algebra, we will always mean a uniformly closed, two-sided ideal.

2. The results. We begin with several propositions that determine the operators which act compactly (respectively weak compactly) on $B(H)$. Throughout, $H$ always denotes a (complex) Hilbert space.

2.1. Proposition. Let $\Phi: B(H) \rightarrow B(H)$ be a bounded linear map which is continuous in the ultraweak operator topology, and maps $K(H)$ into $K(H)$. Then $\varphi=\left(\left.\varphi\right|_{K(H)}\right)^{* *}$, and $\varphi$ is weakly compact if and only if $\varphi(B(H)) \subseteq K(H)$.

† This author was partially supported by NSF grant MCS78-01870

Glasgow Math. J. 21 (1980) 143-149. 
Proof. Note first that $\left(\left.\varphi\right|_{K(H)}\right)^{* *}: B(H) \rightarrow B(H)$ is ultraweakly continuous and agrees with the ultraweakly continuous map $\varphi$ on the ultraweakly dense set $K(H) \subseteq B(H)$, whence $\varphi=\left(\left.\varphi\right|_{K(H)}\right)^{* *}$.

Now assume $\varphi$ is weakly compact. Then $\left(\left.\varphi\right|_{K(H)}\right)^{* *}=\varphi$ is weakly compact, whence $\left.\varphi\right|_{K(H)}$ is weakly compact (Theorem $8,[4$, p. 485] whence $\varphi(B(H)) \subseteq$ norm-closure of $\varphi(K(H)) \subseteq K(H)$ (Theorem 2, [4, p. 482].

Conversely, assume that $\varphi\left(B(H) \subseteq K(H)\right.$. Let $K(H)_{1}$ and $B(H)_{1}$ denote the closed unit balls of $K(H)$ and $B(H)$, respectively. It follows by ultraweak compactness of $B(H)_{1}$ and ultraweak continuity of $\varphi$ that the weak closure of $\varphi\left(K(H)_{1}\right)$ is $\varphi\left(B(H)_{1}\right)$, and this set is $\sigma\left(K(H)^{* *}, K(H)^{*}\right)$-compact. Thus, since $\varphi\left(B(H)_{1}\right) \subseteq K(H)$, and the $\sigma\left(K(H)^{* *}, K(H)^{*}\right)$ topology when restricted to $K(H)$ is the weak topology on $K(H)$, we conclude that the weak closure of $\varphi\left(K(H)_{1}\right)$ is weakly compact.

Q.E.D.

2.2 Proposition. Let $b$ be a nonzero element of $B(H)$ such that any one of the maps

$$
a \rightarrow a b, \quad a \rightarrow b a, \quad a \rightarrow a b+b a, \quad(a \in B(H))
$$

is compact (respectively weakly compact). Then $\operatorname{dim} H<\infty$ (respectively $b \in K(H)$ ).

Proof. The "compact" statement is immediate from [11], the "weakly compact" statement is immediate from Proposition 2.1.

2.3. Proposition. If $b, c \in B(H)$ are both nonzero, then the map $a \rightarrow b a c$ is weakly compact if and only if either $b$ or $c$ is in $K(H)$, and it is compact if and only if both $b$ and $c$ are in $K(H)$.

Proof. Assume $b, c \notin K(H)$. Then by Corollary 5.10 of [3], the ranges of $b$ and $c$ contain closed, infinite-dimensional subspaces. Hence there exists an $a \in B(H)$ which maps a closed, infinite-dimensional subspace of the range of $c$ onto a subspace $M$ of $H$ for which $b(M)$ contains a closed, infinite-dimensional subspace. Thus the range of bac contains a closed, infinite-dimensional subspace, and so by [3], Corollary 5.10, $b a c \notin K(H)$. Thus by Proposition $2.1 a \rightarrow b a c$ is not weakly compact.

Suppose $b \in K(H)$. Then $b a c \in K(H)$ for $a \in B(H)$, so that by Proposition $2.1 a \rightarrow b a c$ is weakly compact.

The statement about compact $a \rightarrow b a c$ is a special case of Theorem 3, p. 174 and Corollary 5, p. 175 of [2]. The proof is complete.

2.4. Lemma. Let $\left\{X_{n}\right\}_{n=1}^{\infty}$ be a sequence of Banach spaces with compact (respectively weakly compact) maps $\varphi_{n}: X_{n} \rightarrow X_{n}$ of uniformly bounded norm. Then $\bigoplus_{n} \varphi_{n}: \bigoplus X_{n} \rightarrow \bigoplus X_{n}$ is compact (respectively weakly compact) if and only if $\lim \left\|\varphi_{n}\right\|=0$. $\left(\oplus X_{n}\right.$ denotes the $l_{\infty}$-direct sum of $\left\{X_{n}\right\}$.)

Proof. We need only verify the weakly compact case, the compact case being an immediate corollary. Suppose with no loss of generality that sup $\left\|\varphi_{n}\right\|=1$. Assume the lemma is false. Since the compression of a weakly compact map to a subspace by a 
continuous projection onto that subspace is weakly compact, we may thus find an $x=\left(x_{n}\right) \in \underset{n}{\bigoplus_{n}} X_{n}$ of norm 1 and a $\delta>0$ such that $\left\|\varphi_{n}\left(x_{n}\right)\right\|>\delta$ for all $n$. Let $M=\hat{\oplus}_{n} X_{n}$. Since $\varphi(x) \notin M$, there is an $f \in X^{*}$ such that $f(\varphi(x))=1$ and $f$ vanishes on $M$.

Define a sequence $\left\{y_{k}=\left(y_{n}^{(k)}\right)\right\} \subseteq \oplus X_{n}$ by

$$
y_{n}^{(k)}=\left\{\begin{array}{l}
0,(n<k) \\
x_{n},(n \geqslant k)
\end{array}\right.
$$

Let $S$ denote the $l_{1}$-direct sum of $\left\{X_{n}^{*}\right\}$. With $S$ actong on $X=\oplus_{n} X_{n}$ in the natural way, we have $S \subseteq X^{*}$, and since $\left\{\left\|\varphi_{n}\right\|\right\}$ is uniformly bounded, $\varphi\left(y_{k}\right) \rightarrow 0$ in the $\sigma(X, S)$-topology. Since the $\sigma(X, S)$-topology is Hausdorff and weaker than the weak topology on $X$, we conclude by weak compactness of $\varphi$ that $\varphi\left(y_{k}\right) \rightarrow 0$ weakly, after perhaps passing to a subsequence and reindexing. But $\varphi\left(y_{k}\right)-\varphi(x) \in M$, for all $k$, and so by the choice of $f$, $f\left(\varphi\left(y_{k}\right)\right)=f(\varphi(x))=1$ for all $k$, a contradiction.

QED

The next result determines the elements of a $\mathrm{C}^{*}$-algebra which act compactly (respectively weak compactly).

2.5. TheOREM. Let $b$ be a nonzero element of a $\mathrm{C}^{*}$-algebra $\mathscr{A}$. Any one of the maps

$$
a \rightarrow a b, \quad a \rightarrow b a, \quad a \rightarrow a b+b a, \quad(a \in \mathscr{A})
$$

is compact if and only if there exists an orthogonal sequence $\left\{p_{n}\right\}$ of minimal, finitedimensional, central projections of $\mathscr{A}$ with $b \in \hat{\oplus}_{n} \mathscr{A} p_{n}$.

Any one of the maps (1) is weakly compact if and only if there exists a sequence $\left\{I_{n}\right\}$ of orthogonal ideals of $\mathscr{A}$ such that each $I_{n}$ is of elementary type and $b \in \underset{n}{\hat{\oplus}} I_{n}$.

Proof. We may pass to the reduced atomic representation of $\mathscr{A}([6, \mathrm{~b}, 35]$ and may hence assume with no loss of generality that $\mathscr{A}$ acts atomically on a Hilbert space $H=\bigoplus_{\alpha} H_{\alpha}$. Let $\mathscr{A}^{-}$denote the closure of $\mathscr{A}$ in the weak operator topology. We have

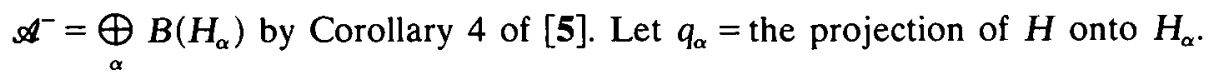

Assume, for instance, that $a \rightarrow a b+b a$ is weakly compact. Arguing as in the proof of Theorem 3.3 of [1], we deduce that $a \rightarrow a b+b a$ is weakly compact on $\mathscr{A}^{-}$and $\left\{x b+b x: x \in \mathscr{A}^{-}\right\} \subseteq \mathscr{A}$. If $b=\oplus b_{\alpha} \in \oplus B\left(H_{\alpha}\right)$, it follows by the proof of Lemma 3.2 of [1] and the fact that the norm of $a \rightarrow a b+b a$ is $2\|b\|$ that all but a countable number of the $b_{\alpha}$ 's, say $b_{\alpha_{n}}=b_{n}$, are zero, and $\lim \left\|b_{n}\right\|=0$ by Lemma 2.4. By Proposition $2.2, b_{n} \in$ $K\left(H_{\alpha_{n}}\right)=K_{n}$, and so $\mathscr{A} \cap K_{n}$ is a nonzero ideal of $\mathscr{A} q_{\alpha_{n}} \supseteq K_{n}$, hence a nonzero ideal of $K_{n}$. Thus $K_{n} \subseteq \mathscr{A}$. We conclude that $b=\oplus b_{n} \in \hat{\oplus} K_{n}$, and the desired result follows. 
If $b=\bigoplus_{n} b_{n} \in \hat{\oplus}_{n} I_{n}$ with $I_{n}$ an ideal of elementary type, by Corollary 4, [4, p. 483], it suffices to show that $a \rightarrow a b_{n}+b_{n} a, a \in \mathscr{A}$, is weakly compact for each $n$. Suppressing the $n$ 's, we may assume with no loss of generality that $b \in I$ is nonnegative. By Proposition 2.3, $a \rightarrow a b^{1 / 2}$ and $a \rightarrow b^{1 / 2} a$ are both weakly compact on $I$, and so $a \rightarrow a b+b a=$ $\left(a b^{1 / 2}\right) b^{1 / 2}+b^{1 / 2}\left(b^{1 / 2} a\right)$ is weakly compact on $\mathscr{A}$ since $b^{1 / 2} a$ and $a b^{1 / 2}$ are in $I$ for all $a \in \mathscr{A}$.

Similar arguments prove the other statements, and so the proof is complete.

In [2] (Definition 9, p. 177), Bonsall and Duncan call a Banach algebra $\mathscr{A}$ compact if for each $b \in \mathscr{A}$, the map $a \rightarrow b a b$ is compact. They show that the Banach algebra of compact operators on a Banach space is compact (Theorem 3(i), [2, p. 177]). Proposition 2.3 and the proof of Theorem 2.5 show that an element $b$ of a $C^{*}$-algebra $\mathscr{A}$ induces a compact map $a \rightarrow b a b$ if and only if $a \rightarrow b a b$ is weakly compact, which happens if and only if $b$ is of the form given in the second part of Theorem 2.5. Hence we immediately deduce the following corollary, which determines the $\mathrm{C}^{*}$-algebras compact in the above sense and which improves on some results of [10] (see also [7]).

2.6. Corollary. Let $\mathscr{A}$ be a $\mathrm{C}^{*}$-algebra. The following are equivalent.

(1) $\mathscr{A}$ is compact in the sense of Bonsall and Duncan.

(2) The map $a \rightarrow b a b, a \in \mathscr{A}$ is weakly compact for each $b \in \mathscr{A}$.

(3) $\mathscr{A}$ is isomorphic to the restricted direct sum of a family of $\mathrm{C}^{*}$-algebras of elementary type.

Moreover, at least one of the maps $a \rightarrow a b, a \rightarrow b a, a \rightarrow a b+b a,(a \in \mathscr{A})$ is compact for each $b \in \mathscr{A}$ if and only if $\mathscr{A}$ is isomorphic to the restricted direct sum of a family of finite-dimensional full matrix algebras.

The next results characterize the ${ }^{*}$-automorphisms of a $\mathrm{C}^{*}$-algebra which are weakly compact perturbations of the identity, but before we state and prove them, the following proposition is needed.

2.7. Proposimon. If $u(\neq 1)$ is a unitary operator in $B(H)$, then $a \rightarrow u a u^{*}-a$ is compact (respectively weakly compact) if and only if $\operatorname{dim} H<\infty$ (respectively $(u+\lambda 1) \in K(H)$ for some complex number $\lambda)$.

Proof. The map $a \rightarrow u a u^{*}-a$ is compact (respectively weakly compact) if and only if the map $a \rightarrow u a-a u$ is the same, since $b \rightarrow b u$ is an isometry of $B(H)$ onto itself. The map $a \rightarrow u a-a u$ is compact (respectively weakly compact) if and only if $\operatorname{dim} H<\infty$ (respectively $(u+\lambda 1) \in K(H)$ for some complex number $\lambda)$ by Lemma 2.1 and Theorem 3.1 of [1].

If $\mathscr{A}$ is a $C^{*}$-algebra, $\operatorname{Aut}(\mathscr{A})$ will denote the group of ${ }^{*}$-automorphisms of $\mathscr{A}$, $\pi=\bigoplus_{\gamma} \pi_{\gamma}: \mathscr{A} \rightarrow B\left(H_{\pi}\right)$ the reduced atomic representation of $\mathscr{A}$. Let $\mathscr{A}_{\pi}=\pi(\mathscr{A}), \mathscr{A}_{\pi}^{-}=$the closure of $\mathscr{A}_{\pi}$ in the weak operator topology in $B\left(H_{\pi}\right)$. We have $H_{\pi}=\underset{\gamma}{\bigoplus_{\gamma}} H_{\gamma}$ where $H_{\gamma}$ is the representation space of $\pi_{\gamma}$, and $\mathscr{A}_{n}^{-}=\bigoplus_{\gamma} B\left(H_{\gamma}\right)$. Let $p_{\gamma}=$ the projection of $H_{\pi}$ onto $H_{\gamma}$, $K_{\gamma}=K\left(H_{\gamma}\right)$. If $\alpha \in \operatorname{Aut}(\mathscr{A}), \alpha_{\pi}$ denotes the ${ }^{*}$-automorphism of $\mathscr{A}_{\pi}$ induced by $\alpha$. 
The following two theorems together determine the structure of *-automorphisms of $\mathscr{A}$ which are weakly compact perturbations of the identity automorphism (denoted id in the sequel) on $\mathscr{A}$.

2.8. Theorem. Let $\mathscr{A}$ be a $\mathrm{C}^{*}$-algebra, $\alpha \in$ Aut $(\mathscr{A})$. The following are equivalent.

(1) $\alpha$-id is weakly compact.

(2) There is a finite-dimensional central projection $p$ of $\mathscr{A}$, an automorphism $\alpha_{1}$ of $p \mathscr{A}$, and an automophism $\alpha_{2}$ of $(1-p) \mathscr{A}$ such that $\alpha_{2}$-id is weakly compact, $\alpha_{2}$ fixes each central element of $(1-p) \mathscr{A}$ and $\alpha=\alpha_{1} \oplus \alpha_{2}$.

Proof. (1) $\Rightarrow(2)$. Let $\mathscr{A}^{* *}$ denote the enveloping von Neumann algebra of $\mathscr{A}$. If $\sigma=\bigoplus\left\{\pi_{f}: f\right.$ a state on $\left.\mathscr{A}\right\}$ denotes the universal representation of $\mathscr{A}$, then by Theorem 1.17.2 of [9], $\mathscr{A}^{* *}$ can be naturally identified with the closure $\sigma(\mathscr{A})^{-}$of $\sigma(\mathscr{A})$ in the weak operator topology.

Let $\alpha \in \operatorname{Aut}(\mathscr{A})$. Since $\alpha^{* *}$ is a ${ }^{*}$-automorphism of $\mathscr{A}^{* *}$ onto $\mathscr{A}^{* *}$, it maps minimal projections onto minimal projections, and, identifying $\mathscr{A}_{\pi}^{-}$in a natural way with the subalgebra of $\mathscr{A}^{* *}$ generated by the minimal projections $([9$, p. 53]), it therefore follows that $\alpha^{* *}\left(\mathscr{A}_{\pi}^{-}\right) \subseteq \mathscr{A}_{\pi}^{-}$. Now assume $\alpha-i d$ is weakly compact.

Since $\alpha^{* *}$ maps minimal central projections onto minimal central projections, it follows that $\alpha^{* *}$ permutes the $p_{\gamma}$ 's in $\mathscr{A}_{\pi}^{-}=\bigoplus_{\gamma} B\left(H_{\gamma}\right)$. Suppose that for $\gamma \neq \lambda, \alpha^{* *}\left(p_{\gamma}\right)=p_{\lambda}$. Consider the map $\varphi: p_{\gamma} \mathscr{A}_{\pi}^{-} \rightarrow p_{\gamma} \mathscr{A}_{\pi}^{-}$defined by $\varphi: a \rightarrow p_{\gamma}\left(\alpha^{* *}(a)-a\right)$. Since $\alpha^{* *}(a) \in p_{\lambda} \mathscr{A}_{\pi}^{-}$, $p_{\gamma}\left(\mathscr{A}^{* *}(a)\right)=0$, whence $\varphi=-i d$ on $p_{\gamma} \mathscr{A}_{\pi}^{-}$. Since $\varphi$ is the composition of a bounded map and the weakly compact map $a \rightarrow \alpha^{* *}(a)-a$, we conclude by Theorem 5 , (4, p. 484], that $\varphi$ is weakly compact, whence $p_{\gamma} \mathscr{A}_{\pi}^{-}$is reflexive, hence finite-dimensional (Proposition 2 of [8]). Thus $\alpha^{* *}$ can only permute finite-dimensional $p_{\gamma}$ 's, and it follows by the weak compactness of $\alpha^{* *}-i d$ and Lemma 2.4 that $\alpha^{* *}$ permutes only a finite number of them.

We want to show next that each $p_{\gamma}$ permuted by $\alpha^{* *}$ is in fact in $\mathscr{A}$. Let $p$ be such a projection, and let $a \in \mathscr{A}$. Then since $p \alpha^{* *}(p)=0$ and $p$ is central,

$$
2 a p+\left(\alpha^{* *}(a p)-a p\right)=\alpha^{* *}(a p)+a p=\left(\alpha^{* *}(a p)-a p\right)\left(\alpha^{* *}(p)-p\right)
$$

But by Theorem 2, [4, p. 482] $\left(\alpha^{* *}-i d\right)\left(\mathscr{A}^{* *}\right) \subseteq \mathscr{A}$. Thus by $(1), a p \in \mathscr{A}$, and so $p \mathscr{A}=$ $\mathscr{A} p \subseteq \mathscr{A}$. Now define $\varphi: a \rightarrow p\left(\alpha^{* *}(a)-a\right)$ as before. Then

$$
-p=\varphi(p) \in \varphi\left(\mathscr{A}^{* *}\right) \subseteq p\left(\alpha^{* *}-i d\right)\left(\mathscr{A}^{* *}\right) \subseteq p \mathscr{A} \subseteq \mathscr{A} \text {. }
$$

Setting $P$ equal to the sum of all the $p_{\gamma}$ 's permuted by $\alpha^{* *}$, we conclude that $P$ is a finite-dimensional central projection in $\mathscr{A}$.

Writing $\mathscr{A}^{* *}=\mathscr{A}^{* *} P \bigoplus \mathscr{A}^{* *}(1-P)$, we have $\alpha^{* *}=\left.\left.\alpha^{* *}\right|_{\mathscr{A} \mathcal{A}^{* *} P} \bigoplus \alpha^{* *}\right|_{a^{* *}(1-P)}$ (notice that $\left.\alpha^{* *}(P)=P\right)$. Since the center of $\mathscr{A}_{\pi}^{-}(1-P)$ is purely atomic and $\left.\alpha^{* *}\right|_{\mathscr{A}_{\pi}^{-}(1-P)}$ fixes each atom, it follows that $\left.\alpha^{* *}\right|_{\mathscr{A}_{\pi}^{*}(1-P)}$ fixes each central element of $\mathscr{A}_{\pi}^{-}(1-P)$. Since $\left.\alpha^{* *}\right|_{\mathscr{A}}=\alpha$, setting $\alpha_{1}=\left.\alpha^{* *}\right|_{\mathscr{A} P}, \alpha_{2}=\left.\alpha^{* *}\right|_{\mathscr{A}(1-P)}$ gives the desired decomposition of $\alpha$.

$(2) \Rightarrow(1)$. This is clear, and so the proof is complete.

2.9. Theorem. Let $\mathscr{A}$ be a $\mathrm{C}^{*}$-algebra, $\alpha \in \operatorname{Aut}(\mathscr{A})$. The following are equivalent.

(1) $\alpha-i d$ is weakly compact and $\alpha$ fixes each central element of $\mathscr{A}$. 
(2) $\alpha_{\pi}$ extends to an inner automorphism $\tilde{\alpha}_{\pi}$ of $\mathscr{A}_{\pi}^{-}$of the following form: there exists a countable set of indices $\left\{\gamma_{n}\right\}$, unitaries $u_{n} \in B\left(H_{\gamma_{n}}\right)$, and complex numbers $\left\{z_{n}\right\}$ such that (if $p_{\gamma}$ is the identity in $B\left(H_{\gamma}\right)$ )

(i) $u_{n}-z_{n} p_{n} \in K_{\gamma_{n}} \subseteq \mathscr{A}_{\pi}$ (where $p_{n}=p_{\gamma_{n}}$ ),

(ii) $\lim \left\|u_{n}-z_{n} p_{n}\right\|=0$,

(iii) $\tilde{\alpha}_{\pi}(a)=u a u^{*},\left(a \in \mathscr{A}_{\pi}^{-}\right)$, where $u=\left(\underset{\gamma \neq \gamma_{n}}{\bigoplus} p_{\gamma}\right) \oplus\left(\bigoplus_{n} u_{n}\right)$.

Proof. (1) $\Rightarrow(2)$. We assert first that $\alpha^{* *} \in \operatorname{Aut}\left(\mathscr{A}^{* *}\right)$ fixes each central element of $\mathscr{A}^{* *}$. By the spectral theorem and $\sigma\left(\mathscr{A}^{* *}, \mathscr{A}^{*}\right)$-continuity of $\alpha^{* *}$, it suffices to show that $\alpha^{* *}(z)=z$ for each central projection $z \in \mathscr{A}^{* *}$. To see this, note first that by Theorem 2 , [4, p. 482], and the weak compactness of $\alpha^{* *}-i d, \alpha^{* *}(z)-z$ is a central element of $\mathscr{A}$. Since $\left.\alpha^{* *}\right|_{\mathscr{A}}=\alpha$ and $\alpha$ fixes each central element of $\mathscr{A}, \alpha^{* *}\left(\alpha^{* *}(z)-z\right)=\alpha^{* *}(z)-z$, i.e.,

$$
\left(\alpha^{* *}\right)^{2}(z)+z=2 \alpha^{* *}(z) \text {. }
$$

Since $\left(\alpha^{* *}\right)^{2}(z), \alpha^{* *}(z)$, and $z$ are projections in an abelian $W^{*}$-algebra (the center of $\mathscr{A}^{* *}$ ), they can be viewed as characteristic functions of measurable sets (Proposition 1.18.1 of [9]), whence by $\left({ }^{*}\right), \alpha^{* *}(z)=z$.

Since $\alpha^{* *}$ fixes each central element, we can apply the reasoning of the proof of Theorem 2.8 to extend $\alpha_{\pi}$ to an automorphism $\tilde{\alpha}_{\pi}$ of $\mathscr{A}_{\pi}^{-}$such that $\bar{\alpha}_{\pi}-i d$ is weakly compact and $\tilde{\alpha}_{\pi}$ fixes each central element of $\mathscr{A}_{\pi}^{-}$. It follows that if $\tilde{\alpha}_{\pi, \gamma}=\left.\tilde{\alpha}_{\pi}\right|_{B\left(H_{\gamma}\right)}$, then $\tilde{\alpha}_{\pi, \gamma} \in \operatorname{Aut}\left(B\left(H_{\gamma}\right)\right), \tilde{\alpha}_{\pi, \gamma}-\left.i d\right|_{B\left(H_{\gamma}\right)}$ is weakly compact, and $\bar{\alpha}_{\pi}=\bigoplus_{\gamma} \tilde{\alpha}_{\pi, \gamma}$. By the proof of Lemma 3.2 of [1], all but a countable number of the $\tilde{\alpha}_{\pi, \gamma}-\left.i d\right|_{B\left(H_{\gamma}\right)}$ 's are nonzero, and if $\left\{\gamma_{n}\right\}$ is the set of the corresponding indices, $\lim _{n}\left\|\tilde{\alpha}_{\pi, \gamma_{n}}-\left.i d\right|_{B\left(H_{\gamma_{n}}\right.}\right\|=0$ by Lemma 2.4. It follows by Proposition 2.7 and the preccding that there exist indices $\left\{\gamma_{n}\right\}$, unitaries $u_{n} \in B\left(H_{\gamma_{n}}\right)$, and complex numbers $\left\{z_{n}\right\}$ satisfying (i), (ii), and (iii).

$(2) \Rightarrow(1)$. This follows easily from Proposition 2.7 and Lemma 2.4 , and so the proof is complete.

\section{REFERENCES}

1. C. A. Akemann and S. Wright, Compact and weakly compact derivations of $\mathrm{C}^{*}$-algebras, Pacific J. Math., to appear.

2. F. F. Bonsall and J. Duncan, Complete normed algebras, Ergebnesse der Mathematik und ihrer Grenzgebiete, Bd. 80, (Springer-Verlag, 1973).

3. R. G. Douglas, Banach algebra techniques in operator theory, (Academic Press, 1972).

4. N. Dunford and J. T. Schwartz, Linear operators, Part I, (Interscience, 1958). $547-556$.

5. J. Glimm and R. V. Kadison, Unitary operators in C*-algebras, Pacific J. Math., 10 (1960),

6. R. V. Kadison and J. R. Ringrose, Derivations and automorphisms of operator algebras, Comm. Math. Phys., 4 (1967), 32-63.

7. T. Ogasawara and K. Yoshinaga, Weakly completely continuous Banach *-algebras, J. Sci. Hiroshima Univ., Ser. A, 18 (1954), 15-36. 
8. S. Sakai, Weakly compact operators on operator algebras, Pacific J. Math., 14 (1964), $659-664$

9. S. Sakai, $\mathrm{C}^{*}$-algebras and $\mathrm{W}^{*}$-algebras, Ergebnesse der Mathematik und ihrer Grenzgebiete, Bd. 60, (Springer-Verlag, 1971).

10. B. J. Tomiuk, Duality and the existence of weakly completely continuous elements in a B*-algebra, Glasgow Math. J., 13 (1972), 56-60. (1967)

11. K. Vala, On compact sets of compact operators, Ann. Acad. Sci. Fenn., Ser. Al, 407

UNIVERSITY OF CALIFORNIA

Santa Barbara

California 93106
OAKLAND University

ROCHESTER

MichigAN 48063 